\title{
Envolvimento Paterno: Construção de um Modelo Teórico Baseado em uma Revisão da Literatura
}

\author{
Ligia de Santis ${ }^{1}$ \\ Elizabeth Joan Barham \\ Universidade Federal de São Carlos, São Carlos, SP, Brasil
}

\begin{abstract}
Resumo
Um corpo amplo de evidências científicas já existente documenta a importância da relação pai-filho. O objetivo deste estudo foi desenvolver um modelo teórico de envolvimento paterno, organizado de acordo com princípios de Análise de Equações Estruturais, que requerem especificar as variáveis associadas ao construto central, com base em dados empíricos. Em 2015, foi realizada uma busca sistemática nas bases de dados eletrônicas APA PsycNET, Bireme, PEPSIC, Web of Science e IndexPsi Periódicos, usando os descritores "envolvimento" e "paterno", cruzados com "avaliação" ou "instrumento", nos idiomas inglês e português. Não foram impostas restrições em relação ao ano de publicação. foram encontrados 39 textos completos que incluíam dados empíricos sobre o envolvimento paterno. As informações encontradas foram agrupadas em três categorias: (a) dimensões do envolvimento paterno, (b) fatores que afetam o envolvimento paterno e (c) impactos deste envolvimento sobre outras variáveis. O modelo apresentado sintetiza os resultados dos estudos e representa um avanço no desenvolvimento teórico na área. Este modelo aumenta a visibilidade de questões conceituais sobre o envolvimento paterno e seus correlatos, e, depois da sua testagem empírica, poderá contribuir para o aprimoramento de programas de intervenção para pais.
\end{abstract}

Palavras-chave: Envolvimento paterno, modelo teórico, desenvolvimento, bem-estar.

\section{Father Involvement: Construction of a Theoretical Model Based on a Literature Review}

\begin{abstract}
An ample body of scientific evidence exists, documenting the importance of the father-child relationship. The objective of this study was to develop a theoretical model of father involvement, organized according to the principles of Structural Equation Modeling, which require specification of the variables associated with the central construct, based on empirical data. In 2015, a systematic search was conducted in the databases APA PsycNET, Bireme, PEPSIC, Web of Science and IndexPsi Periódicos, using the descriptors "involvement" and "father", crossed with "measure", "scale", "tool" or "instrument", in both the English and Portuguese languages. There were no restrictions on the year of publication. In total, 39 complete texts were located, that included data on father involvement. The information in these texts was grouped into three categories: (a) dimensions of father involvement, (b) factors affecting father involvement and (c) impacts of father involvement on other variables. The model presented synthesizes
\end{abstract}

Endereço para correspondência: Universidade Federal de São Carlos, Departamento de Psicologia, Rodovia Washington Luís, Km 235 - SP-310, São Carlos, SP, Brasil 13565-905. Fone: (16) 3351-8361. E-mail: ligiasantis@gmail.com

Reconhecemos o apoio financeiro das agências de fomento: Coordenação de Aperfeiçoamento de Pessoal de Nível Superior (CAPES) e Fundação de Amparo à Pesquisa do Estado de São Paulo (FAPESP), processo ${ }^{\circ}$ 2014/01671-9. 
the results of these studies, representing an advance in theory development in this area. This model increases the visibility of conceptual issues about father involvement and its correlates, and, following empirical testing, may contribute to the improvement of intervention programs for fathers.

Keywords: Father involvement, theoretical model, development, wellbeing.

\section{Envolvimiento Paterno: Construcción de un Modelo Teórico Basado en una Revisión de la Literatura}

\section{Resumen}

Un cuerpo de evidencia científica ya existente documenta la importancia de la relación padre-hijo. El objetivo de este estudio fue desarrollar un modelo teórico de envolvimiento paterno, organizados de acuerdo con los principios de la Análisis de Ecuaciones Estructurales, que requieren especificar las variables asociadas con el constructo central, basada en datos empíricos. En 2015, se realizó una búsqueda sistemática en las bases de datos electrónicas APA PsycNET, Bireme, PEPSIC, Web of Science e IndexPsi Periódicos, utilizando los descriptores "envolvimento" y "paterno", cruzados con "avaliação" o "instrumento", en Inglés y Portugués. No hubo restricciones en el año de publicación. Se encontraron 39 textos completos que incluían datos empíricos sobre el envolvimiento paterno. Estos resultados se agruparon en tres categorías: (a) las dimensiones del envolvimiento paterno, (b) factores que afectan el envolvimiento paterno y (c) el impacto de este envolvimiento en otras variables. El modelo sintetiza los resultados de los estúdios y representa un avance en el desarrollo teórico del área. Este modelo aumenta la visibilidad de las cuestiones conceptuales sobre el envolvimiento paterno y sus correlatos, y, después de su comprobación empírica, puede contribuir a la mejora de los programas de intervención para los padres.

Palabras clave: Envolvimiento paterno, modelo teórico, desarrollo, bienestar.

$\mathrm{O}$ interesse pelo estudo do envolvimento paterno vem aumentando, em especial nas últimas décadas (Volker, 2014). Quando estudos sobre o tema surgiram na literatura acadêmica, os pesquisadores atentavam-se para a presença versus ausência física do pai no ambiente familiar (Cia, 2009), comparando indicadores de desenvolvimento infantil de crianças em ambas as situações. A partir da década de 90, além do foco em interações "face a face" entre o pai e o filho, foram acrescentados os conceitos de acessibilidade (o grau de disponibilidade do pai à criança, tanto física, quanto psicologicamente) e responsabilidade (o quanto o pai assume a responsabilidade pelos cuidados e bem-estar do filho, tomando providências tais como levá-lo ao médico e acompanhar o trabalho de cuidadores pagos (Lamb, 1997; Pleck, 2010). Atualmente, o envolvimento paterno é entendido como sendo um construto multidimensional, abrangendo uma gama de habilidades e englobando dimensões afetivas, cognitivas e éticas, assim como componentes comportamentais observáveis diretos (interações face a face) e indiretos tais como sustento financeiro e suporte psicológico à mãe (Pleck, 2007). Observa-se, portanto, uma recente evolução nos estudos de conceituação do envolvimento paterno.

Apesar desta literatura já existente sobre este conceito, não há um modelo teórico de envolvimento paterno, evidenciando as relações entre este construto e outras variáveis (Pleck, 2007, 2010). Segundo Pasquali (2010), a falta de modelos teóricos sólidos dificulta a construção de ferramentas eficazes para guiar a prática profissional e melhorar a qualidade das pesquisas nesta área (Pleck, 2010).

Um critério para a construção de modelos teóricos científicos é que estes sejam passíveis de testagem empírica. Uma abordagem psicométrica para a criação e avaliação destes modelos teóricos é a dos Modelos de Equações Estruturais (Byrne, 2010). Segundo esta abordagem, inicialmente o pesquisador examina estudos empíricos 
sobre o tema, para obter evidências sobre dimensões do fenômeno de interesse e relações entre este fenômeno e outras variáveis. Em seguida, o pesquisador propõe um modelo que indica prováveis ligações entre estas variáveis, especificando aquelas que influenciam e que são influenciadas pelo fenômeno de interesse (Byrne, 2010; Kline, 2011).

Sendo assim, no presente estudo, tem-se o objetivo de construir um modelo teórico inicial do envolvimento paterno que integre resultados de estudos empíricos que apontam: (a) dimensões do envolvimento paterno, (b) fatores que afetam o envolvimento paterno e (c) impactos do envolvimento paterno.

\section{Método}

A busca bibliográfica foi realizada de fevereiro a março de 2015, nas seguintes bases de dados: APA PsycNET, Bireme, PEPSIC, Web of Science (portal de periódicos CAPES) e IndexPsi Periódicos. Os descritores utilizados, em português, foram: "envolvimento" e "paterno" e "avaliação" ou "instrumento". Em inglês, foram utilizados os descritores: "father" and "involvement" and "measure" or "instrument" or "scale" or "tool". Justifica-se o uso destes descritores pela necessidade de encontrar estudos com evidências empíricas sobre o tema. Não foi imposto limite quanto à data das publicações e foram considerados para análise apenas artigos (uma vez que estes já passaram por uma avaliação de pares), sendo que o artigo deveria estar disponível na íntegra. Além destes critérios, as informações empíricas reportadas nos artigos tinham que abordar um ou mais dos seguintes temas: (a) dimensões do envolvimento paterno, discutidas em artigos sobre a criação ou validação de instrumentos psicométricos acerca do envolvimento paterno, (b) fatores que afetam o envolvimento paterno e (c) impactos deste envolvimento sobre outras variáveis. A análise dos artigos foi feita por duas pesquisadoras, que examinaram os trabalhos e entraram em consenso (Anfara, Brown, \& Mangione, 2002) para extrair as informações relevantes para cada categoria.
Artigos encontrados em mais de uma base de dados foram contabilizados apenas uma vez. Dos 95 artigos encontrados, 41 foram excluídos por terem apenas o resumo acessível. Durante o processo de categorização, 15 artigos foram excluídos por não conterem resultados empíricos que se encaixassem nestas categorias.

\section{Resultados}

A apresentação dos resultados será feita para cada categoria definida nos objetivos do estudo: (a) Dimensões do envolvimento paterno, (b) Fatores que afetam o envolvimento paterno e (c) Impactos do envolvimento paterno.

\section{Dimensões do Envolvimento Paterno}

Para contribuir com a construção de uma teoria unificada sobre envolvimento paterno, é de central importância verificar a forma como diferentes pesquisadores entendem e operacionalizam este conceito. Assim, agruparam-se os sete artigos que tinham como objetivo a criação ou avaliação de instrumentos psicométricos para medir o envolvimento paterno, verificando-se como o construto foi abordado em cada um deles. Na Tabela 1, são apresentas informações sobre esses artigos.

Wysocki e Gavin (2004), assim como outros autores, destacaram a importância de considerar tanto a quantidade, quanto a qualidade do envolvimento paterno (Finley \& Schwartz, 2004; Gomez \& Leal, 2007). Por exemplo, Finley e Schwartz (2004) e Gomez e Leal (2007) analisaram a qualidade afetiva da relação pai-filho ou das experiências vivenciadas pelo pai em relação ao filho.

Além disso, o envolvimento paterno pode ser dividido em atividades "diretas", face a face com a criança, e "indiretas", em prol da criança. Em relação ao envolvimento direto do pai, Escribano et al. (2013) examinaram práticas parentais, levando em consideração aspectos como disciplina e supervisão. Finley e Schwartz (2004) também investigaram o envolvimento direto (como em relação às tarefas escolares do filho) e, além disso, o envolvimento paterno indireto (como prover renda para a família). 
Tabela 1

Descrição dos Estudos Agrupados na Categoria "Dimensões do Envolvimento Paterno".

\begin{tabular}{|c|c|c|c|c|}
\hline Referência & País & Amostra & $\begin{array}{l}\text { Idade } \\
\text { do } \\
\text { filho }\end{array}$ & Dimensões do envolvimento paterno \\
\hline $\begin{array}{l}\text { Wysocki \& Gavin } \\
\qquad(2004)\end{array}$ & EUA & $\begin{array}{l}224 \text { casais } \\
\text { (pai e mãe) } \\
\text { com filho doente }\end{array}$ & $\begin{array}{c}2-18 \\
\text { anos }\end{array}$ & $\begin{array}{l}\text { Quantidade de envolvimento paterno; Contribuição } \\
\text { do envolvimento paterno }\end{array}$ \\
\hline $\begin{array}{l}\text { Finley \& Schwartz } \\
\qquad(2004)\end{array}$ & EUA & $\begin{array}{l}2.353 \text { homens } \\
\text { e mulheres }\end{array}$ & $\mathrm{R} *$ & $\begin{array}{l}\text { Medida 1: Qualidade afetiva do envolvimento paterno } \\
\text { Medida 2: Envolvimento emocional, social, espiritual; } \\
\text { Envolvimento financeiro, ético, responsável, protetivo; } \\
\text { Ensinar condutas }\end{array}$ \\
\hline $\begin{array}{l}\text { Gomez \& Leal } \\
\quad(2007)\end{array}$ & Portugal & $\begin{array}{l}105 \text { pais e } 107 \\
\text { mães durante } \\
\text { a gravidez }\end{array}$ & $\mathrm{G}^{* *}$ & Vinculação pré-natal \\
\hline $\begin{array}{l}\text { Escribano, Aniorte, } \\
\text { \& Orgilés (2013) }\end{array}$ & Espanha & 423 filhos & $\begin{array}{l}8-12 \\
\text { anos }\end{array}$ & $\begin{array}{l}\text { Envolvimento parental; Envolvimento positivo; } \\
\text { Disciplina inconsistente; Supervisão insuficiente }\end{array}$ \\
\hline Dick (2004) & EUA & 311 homens & $\mathrm{R}^{*}$ & $\begin{array}{l}\text { Engajamento positivo; Responsividade emocional } \\
\text { positiva; Engajamento negativo; Papel moral do pai, } \\
\text { Papel de bom provedor; Modelo de gênero; Pai não } \\
\text { tradicional (Androgynous Role); Acessibilidade; } \\
\text { Responsabilidade }\end{array}$ \\
\hline $\begin{array}{l}\text { Halme, Tarkka, } \\
\text { Paavilainen, } \\
\text { Nummi, \& } \\
\text { Åstedt-Kurki (2010) }\end{array}$ & Finlândia & 1.084 pais & $\begin{array}{l}3-6 \\
\text { anos }\end{array}$ & $\begin{array}{l}\text { Atividades compartilhadas entre pai e criança; } \\
\text { Prazer no envolvimento; Envolvimento potencial; } \\
\text { Conflitos diários relacionados ao envolvimento }\end{array}$ \\
\hline $\begin{array}{l}\text { Ly \& Goldberg } \\
\qquad(2014)\end{array}$ & USA & $\begin{array}{c}101 \text { pais de } \\
\text { crianças com } \\
\text { desenvolvimento } \\
\text { atípico }\end{array}$ & $\begin{array}{c}2-17 \\
\text { anos }\end{array}$ & $\begin{array}{l}\text { Impacto sobre a paternidade; Envolvimento } \\
\text { com a intervenção da criança }\end{array}$ \\
\hline
\end{tabular}

Notas. * A letra "R" significa que o estudo é retrospectivo; ou seja, os participantes descreviam relações que tiveram no passado com seus pais. **Estudo aconteceu durante a gestação do filho; ou seja, o filho ainda não havia nascido.

Em três dos trabalhos encontrados (Dick, 2004; Halme et al., 2010; Ly \& Goldberg, 2014) o envolvimento paterno foi operacionalizado com base nos conceitos propostos por Michel Lamb (1997): interação (envolvimento direto), acessibilidade e responsabilidade (envolvimento indireto).

Outros estudiosos basearam-se em mais de um pressuposto teórico para construir seus instrumentos. Estes autores, além de abordarem a definição de Lamb (1997), também avaliaram a dimensão do envolvimento paterno. Ly e Goldber (2014), por exemplo, examinaram o envolvimento cognitivo. Dick (2004), Finley e Schwartz (2004) e Gomez e Leal (2007), por sua vez, investigaram o envolvimento emocional.
Por fim, Dick (2004) verificou as funções sociais paternas (ou seja, as funções do pai em relação à adaptação social do filho, envolvendo questões morais, de gênero e financeiras).

\section{Fatores que Afetam o Envolvimento Paterno}

Nos estudos sobre o envolvimento paterno, vários autores analisaram evidências acerca de condições que poderiam influenciar no relacionamento entre pai e filho. Na Tabela 2 são apresentadas algumas informações sobre estes 15 estudos. Nota-se que estes fatores são de diferentes naturezas, podendo envolver condições de vida ou habilidades apresentadas pelos pais, pelas mães ou por ambos. 
da Literatura.

Tabela 2

Descrição dos Estudos Agrupados na Categoria “Fatores que Afetam o Envolvimento Paterno".

\begin{tabular}{|c|c|c|c|c|}
\hline Referência & País & Amostra & $\begin{array}{l}\text { Idade } \\
\text { do filho }\end{array}$ & $\begin{array}{c}\text { Fatores que afetam } \\
\text { o envolvimento paterno }\end{array}$ \\
\hline Silva \& Aiello (2009) & Brasil & $\begin{array}{l}13 \text { pais de crianças com } \\
\text { deficiência mental }\end{array}$ & $\begin{array}{l}2-4 \\
\text { anos }\end{array}$ & Pai: escolaridade \\
\hline $\begin{array}{l}\text { Brandão \& Figueiredo } \\
\text { (2012) }\end{array}$ & Portugal & 105 pais & $\begin{array}{l}0-1 \\
\text { mês }\end{array}$ & $\begin{array}{l}\text { Pai: envolvimento prático no momento } \\
\text { do nascimento do filho }\end{array}$ \\
\hline Cia \& Barham (2006) & Brasil & 58 pais & $\begin{array}{l}10-14 \\
\text { anos }\end{array}$ & $\begin{array}{l}\text { Pai: turno e número de horas } \\
\text { de trabalho }\end{array}$ \\
\hline $\begin{array}{l}\text { Warpechowski \& } \\
\text { Mosmann (2012) }\end{array}$ & Brasil & $\begin{array}{l}3 \text { pais que enfrentaram } \\
\text { separação conjugal }\end{array}$ & $\begin{array}{l}3-16 \\
\text { anos }\end{array}$ & Pai: estatus conjugal \\
\hline Broger \& Zeni (2011) & EUA & $\begin{array}{l}54 \text { pais de filhos com } \\
\text { doença crônica }\end{array}$ & $\begin{array}{l}\text { Até } 21 \\
\text { anos }\end{array}$ & $\begin{array}{l}\text { Pai: estatus conjugal, idade } \\
\text { e escolaridade }\end{array}$ \\
\hline $\begin{array}{l}\text { Grzybowski \& Wagner } \\
\text { (2010) }\end{array}$ & Brasil & $\begin{array}{c}234 \text { pais e mães separa- } \\
\text { dos / divorciados }\end{array}$ & $\begin{array}{l}6-12 \\
\text { anos }\end{array}$ & $\begin{array}{l}\text { Pai: coabitação com o filho, } \\
\text { escolaridade, tipo de ocupação; } \\
\text { Relação pai-mãe: qualidade } \\
\text { da relação conjugal }\end{array}$ \\
\hline $\begin{array}{l}\text { Habib \& Lancaster } \\
\qquad(2010)\end{array}$ & Austrália & $\begin{array}{l}78 \text { pais do primeiro } \\
\text { filho }\end{array}$ & $\mathrm{G}^{*}$ & $\begin{array}{l}\text { Pai: envolvimento emocional durante } \\
\text { a gestação }\end{array}$ \\
\hline $\begin{array}{l}\text { Van Ee, Sleijpen, } \\
\text { Kleber, \& Jongmans } \\
\text { (2013) }\end{array}$ & Holanda & $\begin{array}{l}80 \text { pais com indícios } \\
\text { de trauma }\end{array}$ & $\begin{array}{l}18-42 \\
\text { meses }\end{array}$ & $\begin{array}{l}\text { Pai: saúde mental (estresse } \\
\text { pós-traumático) }\end{array}$ \\
\hline $\begin{array}{l}\text { Cauce, Conger, Cruz, } \\
\text { King, \& Widaman } \\
(2011)\end{array}$ & EUA & $\begin{array}{l}450 \text { filhos de famílias } \\
\text { biparentais de origem } \\
\text { mexicana }\end{array}$ & $5^{\mathrm{a}}$ série & Pai: adesão às normas culturais \\
\hline $\begin{array}{l}\text { Shapiro, Krysik, \& } \\
\quad \text { Pennar (2011) }\end{array}$ & EUA & $\begin{array}{c}197 \text { mães cujas famílias } \\
\text { estavam em risco para } \\
\text { o abuso infantil }\end{array}$ & 3 meses & $\begin{array}{l}\text { Pai: capacidade para lidar com tarefas } \\
\text { financeiras }\end{array}$ \\
\hline Waller \& Bitler (2008) & EUA & 3.103 pais e mães & $\begin{array}{l}\text { Recém } \\
\text { nascido }\end{array}$ & $\begin{array}{l}\text { Pai: intenção de aborto do pai ou da } \\
\text { mãe (não casados) }\end{array}$ \\
\hline $\begin{array}{l}\text { Murray, Rosengard, } \\
\text { Weitzen, Raker, } \\
\text { \& Phipps (2012) }\end{array}$ & EUA & $\begin{array}{l}300 \text { mães adolescentes } \\
(12-19 \text { anos }) \text { durante } \\
\text { a gravidez }\end{array}$ & $\mathrm{G}^{*}$ & $\begin{array}{l}\text { Pai: apoio à mãe durante a gestação } \\
\text { e envolvimento prático no nascimento } \\
\text { do filho; } \\
\text { Mãe: características sociodemográficas }\end{array}$ \\
\hline $\begin{array}{l}\text { Falceto, Fernandes, } \\
\text { Baratojo, \& Giugliani } \\
\text { (2008) }\end{array}$ & Brasil & 153 famílias & 4 meses & $\begin{array}{l}\text { Mãe: se exerce trabalho remunerado } \\
\text { Relação pai-mãe: qualidade } \\
\text { da relação conjugal }\end{array}$ \\
\hline $\begin{array}{l}\text { Ehrenberg, Gearing- } \\
\text {-Small, Hunter, \& } \\
\text { Small (2001) }\end{array}$ & Canadá & $\begin{array}{l}58 \text { casais em que pai } \\
\text { e mãe exercem } \\
\text { trabalho remunerado }\end{array}$ & $\begin{array}{l}\text { Até } 9 \\
\text { anos }\end{array}$ & $\begin{array}{l}\text { Relação pai-mãe: parentalidade } \\
\text { compartilhada }\end{array}$ \\
\hline $\begin{array}{l}\text { Formoso, Gonzales, } \\
\text { Barrera, \& Dumka } \\
\text { (2007) }\end{array}$ & EUA & $\begin{array}{l}115 \text { famílias biparentais } \\
\text { de origem mexicana }\end{array}$ & $\begin{array}{l}11-14 \\
\text { anos }\end{array}$ & $\begin{array}{l}\text { Relação pai-mãe: qualidade da relação } \\
\text { conjugal; aliança entre pai e mãe }\end{array}$ \\
\hline
\end{tabular}

Nota. *A letra "G" significa que o estudo aconteceu durante a gestação do filho; ou seja, o filho ainda não havia nascido. 
Fatores Ligados ao Pai. Estudiosos avaliaram a influência de características paternas sobre o relacionamento pai-filho. Broger e Zeni (2011), por exemplo, verificaram que pais casados apresentam um melhor envolvimento com seus filhos, em comparação com aqueles que não coabitavam com estes (Grzybowski \& Wagner, 2010). Em relação aos pais divorciados, Warpechowski e Mosmann (2012) verificaram que estes pais relataram um desejo de maior participação na vida dos filhos.

O nível de escolaridade (Grzybowski \& Wagner, 2010; Silva \& Aiello, 2009) e a idade (Broger \& Zeni, 2011) do pai também apareceram como correlatos do envolvimento paterno; quanto maior a idade e a escolaridade do pai, melhor seu envolvimento com o filho. Além disso, quanto maior a capacidade do pai para lidar com tarefas financeiras (representada pela renda familiar mensal e pela posse de carros), maior o nível de envolvimento paterno (Shapiro et al., 2011). Em relação à saúde mental do pai, van Ee et al. (2013) apontaram a influência negativa do estresse pós-traumático sobre a qualidade da relação pai-filho.

Além destas características, alguns pesquisadores investigaram a influência do trabalho remunerado do pai sobre o envolvimento paterno, mostrando que o tipo de ocupação (Grzybowski \& Wagner, 2010) e o turno e número de horas de trabalho do pai (Cia \& Barham, 2006) afetam o envolvimento paterno: quanto mais horas o pai passa na companhia do seu filho, melhor seu envolvimento.

O envolvimento do pai com o filho inicia no período gestacional (Piccinini, Silva, Gonçalves, Lopes, \& Tudge, 2004). Quanto mais intenso o envolvimento emocional do pai durante o período de gestação (Habib \& Lancaster, 2010) e quanto maior o apoio à mãe (Murray et al., 2012) e o envolvimento prático do pai no momento do nascimento do filho (Brandão \& Figueiredo, 2012; Murray et al., 2012), maiores as chances do pai se envolver em atividades paternas no início da vida do seu filho. O relato da intenção de aborto do pai ou da mãe (não casados) também pareceu influenciar os comportamentos parentais durante a gravidez, diminuindo a probabili- dade do pai ajudar com custos durante a gestação (Waller \& Bitler, 2008).

Por fim, Cauce et al. (2011) examinaram a influência de normas culturais sobre a relação pai-filho. Segundo eles, a adesão do pai às normas culturais em relação ao papel do homem e suas responsabilidades dentro da família estava associada com percepções positivas dos filhos sobre o envolvimento paterno. Ou seja, o envolvimento é bem avaliado quando o pai segue as normas culturais vigentes.

Fatores Ligados às Mães. Murray et al. (2012) mostraram que características sociodemográficas das mães (tais como, raça, idade e país de nascimento), estão relacionadas com um aumento na probabilidade do pai registrar o nascimento do seu filho, o que é entendido como um primeiro passo para o pai assumir um envolvimento a longo prazo. Falceto et al. (2008), por sua vez, observaram que, quando as mães não exerciam trabalho remunerado, existia uma probabilidade menor do pai se envolver com o filho, do que quando a mãe era economicamente ativa.

Fatores Ligados à Relação entre o Pai e Mãe da Criança. Falceto et al. (2008), Formoso et al. (2007) e Grzybowski e Wagner (2010) encontraram que relações conjugais desgastadas estão relacionadas com o baixo envolvimento paterno. De forma complementar, a existência de aliança entre pai e mãe influenciou positivamente a qualidade do relacionamento pai-filho (Formoso et al., 2007). Além disso, a qualidade da parentalidade compartilhada (processo interparental de cooperação e suporte mútuo) também se mostrou positivamente relacionada com o envolvimento paterno (Ehrenberg et al., 2001).

\section{Impactos do Envolvimento Paterno}

Além das evidências sobre fatores que afetam o envolvimento paterno, em 19 artigos foram apresentados resultados que apontam para possíveis impactos deste envolvimento (ver Tabela $3)$. Alguns dos impactos investigados dizem respeito aos filhos, suas mães, seus pais ou a família como um todo. Em cada um desses casos, há resultados sobre diferentes áreas da vida da pessoa. 
Tabela 3

Descrição dos Estudos Agrupados na Categoria “Impactos do Envolvimento Paterno".

\begin{tabular}{|c|c|c|c|c|}
\hline Referência & País & Amostra & $\begin{array}{l}\text { Idade } \\
\text { do filho }\end{array}$ & Impactos do envolvimento paterno \\
\hline $\begin{array}{l}\text { Ramchandani et al. } \\
\text { (2013) }\end{array}$ & $\begin{array}{l}\text { Reino } \\
\text { Unido }\end{array}$ & 192 famílias & 1 ano & Filho: comportamentos externalizantes \\
\hline $\begin{array}{l}\text { Cia, Barham, } \\
\& \text { Fontaine }(2010)\end{array}$ & Brasil & $\begin{array}{l}20 \text { professores } \\
\text { e } 99 \text { filhos (pais } \\
\text { participaram de } \\
\text { uma intervenção) }\end{array}$ & $\begin{array}{l}8 \text { anos } \\
(\text { em } \\
\text { média })\end{array}$ & $\begin{array}{l}\text { Filho: comportamentos internalizantes } \\
\text { e externalizantes }\end{array}$ \\
\hline Cia \& Barham (2009) & Brasil & $\begin{array}{c}97 \text { casais } \\
\text { (pais e mães) e } \\
20 \text { professores }\end{array}$ & $\begin{array}{l}6-9 \\
\text { anos }\end{array}$ & $\begin{array}{l}\text { Filho: hiperatividade, comportamentos } \\
\text { internalizantes e externalizantes }\end{array}$ \\
\hline $\begin{array}{l}\text { Bronte-Tinkew, } \\
\text { Moore, Capps, } \\
\text { \& Zaff }(2006)\end{array}$ & EUA & $\begin{array}{l}7242 \text { filhos } \\
\text { (adolescentes) }\end{array}$ & $\begin{array}{l}\text { Até } 18 \\
\text { anos }\end{array}$ & Filho: comportamentos externalizantes \\
\hline $\begin{array}{l}\text { Freeze, Burke, \& } \\
\text { Vorster (2014) }\end{array}$ & $\begin{array}{l}\text { África } \\
\text { do Sul }\end{array}$ & $\begin{array}{l}80 \text { filhos } \\
\text { (meninos) }\end{array}$ & $\begin{array}{l}14-18 \\
\text { anos }\end{array}$ & Filho: comportamentos externalizantes \\
\hline $\begin{array}{l}\text { Cia, Barham, \& } \\
\text { Fontaine }(2012)\end{array}$ & Brasil & $\begin{array}{c}97 \text { casais } \\
\text { (pais e mães) }\end{array}$ & $\begin{array}{l}6-9 \\
\text { anos }\end{array}$ & $\begin{array}{c}\text { Filho: desempenho acadêmico } \\
\text { e autoconceito }\end{array}$ \\
\hline $\begin{array}{l}\text { Falco, Esposito, } \\
\text { Venuti, \& Bornstein } \\
\qquad(2008)\end{array}$ & Itália & 19 filhos & $\begin{array}{c}35.32 \\
\text { meses } \\
\text { (em média) }\end{array}$ & $\begin{array}{l}\text { Filho: aparecimento do comportamento } \\
\text { simbólico }\end{array}$ \\
\hline Salonna et al. (2012) & Eslováquia & 1.863 filhos & $\begin{array}{l}16.85 \text { anos } \\
\text { (em média) }\end{array}$ & Filho: saúde geral \\
\hline $\begin{array}{c}\text { Uji, Sakamoto, } \\
\text { Adachi, \& Kitamura } \\
\text { (2014) }\end{array}$ & Japão & 1.320 adultos & $\mathrm{R}^{*}$ & $\begin{array}{l}\text { Filho: medidas de depressão, ansiedade, } \\
\text { sintomas físicos, funcionamento social } \\
\text { e bem-estar psicológico }\end{array}$ \\
\hline Formoso et al. (2007) & EUA & $\begin{array}{l}115 \text { famílias } \\
\text { biparentais de } \\
\text { origem mexicana }\end{array}$ & $\begin{array}{c}11-14 \\
\text { anos }\end{array}$ & $\begin{array}{l}\text { Filho: saúde mental (depressão), } \\
\text { comportamentos externalizantes }\end{array}$ \\
\hline $\begin{array}{l}\text { Cerniglia, Cimino, \& } \\
\text { Ballarotto (2014) }\end{array}$ & Itália & 77 famílias & $\begin{array}{c}23-26 \\
\text { meses }\end{array}$ & $\begin{array}{l}\text { Filho: qualidade da relação pai-filho durante } \\
\text { as refeições, quando o pai o alimentava }\end{array}$ \\
\hline Tessier et al. (2009) & Colômbia & 338 famílias & 1 ano & $\begin{array}{l}\text { Filho: desenvolvimento locomotor, social, } \\
\text { da fala, da audição e da coordenação motora; } \\
\text { Família: estimulação ambiental, abertura social } \\
\text { e positividade das interações mãe-filho }\end{array}$ \\
\hline $\begin{array}{c}\text { Séjourné, Beaumé, } \\
\text { Vaslot, \& Chabrol } \\
\text { (2012) }\end{array}$ & França & $\begin{array}{l}51 \text { casais } \\
\text { (mãe e pai) }\end{array}$ & $0-4$ meses & Mãe: depressão pós-parto \\
\hline Fagan \& Lee (2010) & EUA & $\begin{array}{l}100 \text { mães } \\
\text { adolescentes }\end{array}$ & 0 - 3 meses & Mãe: depressão pós-parto \\
\hline $\begin{array}{l}\text { Séjourné, Vaslot, } \\
\text { Beaumé, Goutaudier, } \\
\text { \& Chabrol (2012) }\end{array}$ & França & $\begin{array}{l}119 \text { casais } \\
\text { (mãe e pai) }\end{array}$ & $0-2$ meses & Mãe: depressão pós-parto \\
\hline $\begin{array}{l}\text { Alexander, } \\
\text { O’Riordan, \& } \\
\text { Furman }(2010)\end{array}$ & EUA & $\begin{array}{l}176 \text { grávidas } \\
\text { de baixa renda }\end{array}$ & $\mathrm{G}^{* *}$ & $\begin{array}{l}\text { Mãe: intenção e envolvimento } \\
\text { na amamentação }\end{array}$ \\
\hline
\end{tabular}




\begin{tabular}{|c|c|c|c|c|}
\hline Referência & País & Amostra & $\begin{array}{l}\text { Idade } \\
\text { do filho }\end{array}$ & Impactos do envolvimento paterno \\
\hline $\begin{array}{l}\text { Shapiro et al. } \\
\qquad(2011)\end{array}$ & EUA & $\begin{array}{l}197 \text { mães cujas } \\
\text { famílias estavam } \\
\text { em risco para o } \\
\text { abuso infantil }\end{array}$ & 3 meses & $\begin{array}{l}\text { Mãe: percepção de solidão; } \\
\text { Família: cuidados pré-natais, casos de violência } \\
\text { física doméstica e renda familiar }\end{array}$ \\
\hline $\begin{array}{l}\text { Bonner, Hardy, } \\
\text { Willard, \& } \\
\text { Hutchinson (2007) }\end{array}$ & EUA & $\begin{array}{l}23 \text { pais (cuida- } \\
\text { dores primários } \\
\text { de um filho com } \\
\text { doença crônica) }\end{array}$ & $\begin{array}{l}9.5 \text { anos } \\
\text { (em média) }\end{array}$ & Pai: sofrimento psicológico e depressão \\
\hline $\begin{array}{l}\text { Frascarolo, } \\
\text { Zaouche-Gaudron, } \\
\text { \& Favez (2005) }\end{array}$ & Suíça & $\begin{array}{l}13 \text { pais do } \\
\text { primeiro filho }\end{array}$ & $\begin{array}{l}0-18 \\
\text { meses }\end{array}$ & Família: aliança familiar \\
\hline
\end{tabular}

Nota. * Estudo é retrospectivo; ou seja, os participantes descreviam relações que tiveram no passado com seus pais. ** Estudo aconteceu durante a gestação do filho; ou seja, o filho ainda não havia nascido.

Impactos do Envolvimento Paterno sobre os Filhos. Bronte-Tinkew et al. (2006), Cia e Barham (2009), Cia, Barham e Fontaine (2010), Formoso et al. (2007), Freeze et al. (2014) e Ramchandani et al. (2013), encontraram uma relação negativa entre a qualidade do envolvimento paterno e a ocorrência ou frequência das seguintes dificuldades por parte do filho: (a) problemas de saúde mental (como depressão), (b) comportamentos externalizantes, (c) comportamentos internalizantes ou (d) hiperatividade. Neste sentido, Uji et al. (2014) relataram que crianças com pais autoritários (com qualidade menor de envolvimento) apresentaram piores resultados em medidas de depressão, ansiedade, sintomas físicos, funcionamento social e bem-estar psicológico.

Além destes impactos, o baixo envolvimento paterno estava associado com indicadores de menor desempenho acadêmico e autoconceito do filho (Cia, Barham, \& Fontaine, 2012). Falco et al. (2008), por sua vez, verificaram que o envolvimento positivo dos pais favorecia o aparecimento do comportamento simbólico entre crianças com síndrome de Down. Outras consequências positivas de um envolvimento paterno mais frequente incluíram autorrelatos acerca da saúde geral dos filhos (Salonna et al., 2012) e sobre a qualidade da relação pai-filho durante as refeições, quando o pai o alimentava (Cerniglia et al., 2014). Tessier et al. (2009), por sua vez, encontraram que quando o envolvimento paterno era classificado como "alto", este tinha um efeito positivo sobre o desenvolvimento dos filhos do sexo masculino nas seguintes áreas: locomotora, social, fala, audição e coordenação motora.

Impactos do Envolvimento Paterno sobre as Mães. Pesquisadores observaram que quanto menor o envolvimento paterno ou a satisfação materna com este envolvimento, mais intensos os sintomas de depressão pós-parto materna (Fagan, \& Lee, 2010; Séjourné, Beaumé, et al., 2012; Séjourné, Vaslot, et al., 2012) e maior a percepção de solidão materna (Shapiro et al., 2011). Além disso, Alexander et al. (2010) estudaram expectativas sobre o período de amamentação, encontrando que o apoio do pai influenciou significativamente na intenção e no posterior envolvimento da mãe na amamentação.

Impactos do Envolvimento Paterno sobre os Pais. Encontrou-se apenas um estudo sobre os efeitos do envolvimento paterno para o próprio pai. Bonner et al. (2007) observaram que, quanto maior o envolvimento paterno com um filho com câncer, maior o sofrimento psicológico e depressão entre os pais. Não houve estudos sobre pais de filhos com desenvolvimento típico.

Impactos do Envolvimento Paterno sobre a Família. Tessier et al. (2009) verificaram que famílias com pais mais envolvidos foram avaliadas como oferecendo ambientes mais estimulantes, mais abertas a outras pessoas (tais como a famí- 
lia extensa e vizinhos) e com interações mãe-filho mais positivas. Frascarolo et al. (2005), por sua vez, observaram alianças familiares mais fortes quando o pai era mais participativo. Em outro estudo, Shapiro et al. (2011) encontraram associações entre o envolvimento positivo do pai e: (a) melhores cuidados pré-natais durante a gravidez, (b) menor número de casos de violência física doméstica e (c) maior renda familiar.

\section{Discussão}

Esta revisão deixa clara a amplitude das evidências empíricas que indicam a relação entre o envolvimento paterno e outras variáveis. Por meio das informações apresentadas, é possível observar variações em relação a quem avalia o envolvimento paterno, além de diferenças em termos de faixa etária, estatus conjugal e condições de saúde dos pais, mães e filhos, por exemplo. Variações na composição da amostra entre os estudos podem ajudar a elucidar a relação entre o envolvimento paterno e outras variáveis, contribuindo para a construção de um modelo teórico inicial acerca do envolvimento paterno, baseado em evidências. Desta forma, os resultados encontrados foram organizados para examinar e integrar informações acerca de: (a) conceitos sobre o envolvimento paterno, (b) fatores que afetam o envolvimento paterno e (c) impactos do envolvimento paterno.

Os resultados desta revisão da literatura apresentam semelhanças com os do estudo de Gomes, Bossardi, Cruz, Crepaldi e Vieira (2014), no qual foram comparados instrumentos de avaliação do envolvimento paterno. Estes pesquisadores analisaram 15 artigos científicos e verificaram que nestes também destacava-se a importância dos três conceitos de Lamb (1997; interação, acessibilidade e responsabilidade), que fundamentaram a maior parte dos instrumentos. Os autores relataram que os instrumentos também foram construídos para avaliar dimensões mais específicas da relação pai-filho, como o envolvimento emocional (afetivo), as práticas parentais ou envolvimento direto (disciplina e envolvimento didático) e a comunicação. Além disso, os autores apontaram a possibilidade de avaliar estas dimensões em termos da qualidade e quantidade do engajamento do pai. No presente estudo, além de encontrar conceitos adicionais para definir o envolvimento paterno (tais como as dimensões cognitiva e social e a explicitação do envolvimento indireto), foram agregadas informações sobre outras variáveis que afetam ou são afetadas pelo envolvimento do pai.

As informações encontradas em uma revisão sistemática da literatura dependem dos descritores escolhidos e das questões investigadas pelos pesquisadores que conduziram os estudos encontrados. Por exemplo, nos resultados desta revisão não foram encontrados trabalhos que avaliassem o envolvimento paterno com base na dimensão de estimulação física. Segundo Manfroi, Macarini e Vieira (2011), o tipo de interação pai-criança mais frequente é a brincadeira turbulenta (dimensão física do envolvimento), sendo esta importante para o desenvolvimento do filho. Além disso, notou-se que características dos filhos (como suas habilidades sociais) não apareceram, neste estudo, como fatores que poderiam modificar o envolvimento paterno, apesar de informações desta natureza aparecerem em outros trabalhos (Mehall, Spinrad, Eisenberg, \& Gaertner, 2009; Volker, 2014). O não aparecimento destas informações pode estar ligado aos descritores utilizados neste estudo de revisão, já que alguns autores podem ter usado descritores mais gerais sobre a relação pai-filho, ou usado outros descritores (por exemplo, engajamento paterno). Assim, a fim de apresentar um modelo teórico mais completo, estas duas variáveis foram incluídas em caixas tracejadas, na Figura 1 , juntamente com as informações descritas nos resultados deste estudo.

Conforme mostrado na Figura 1, o envolvimento paterno é apresentado como uma variável interveniente, que modifica os impactos das condições e habilidades pré-existentes dos pais e mães sobre o desenvolvimento ou bem- estar da criança, do pai ou da mãe. Em relação aos fatores que influenciam no envolvimento paterno, observa-se um foco nas características pessoais, sociais e culturais de pais e mães e no relacionamento entre ambos, o que está em acordo com conceitos apresentados por outros 


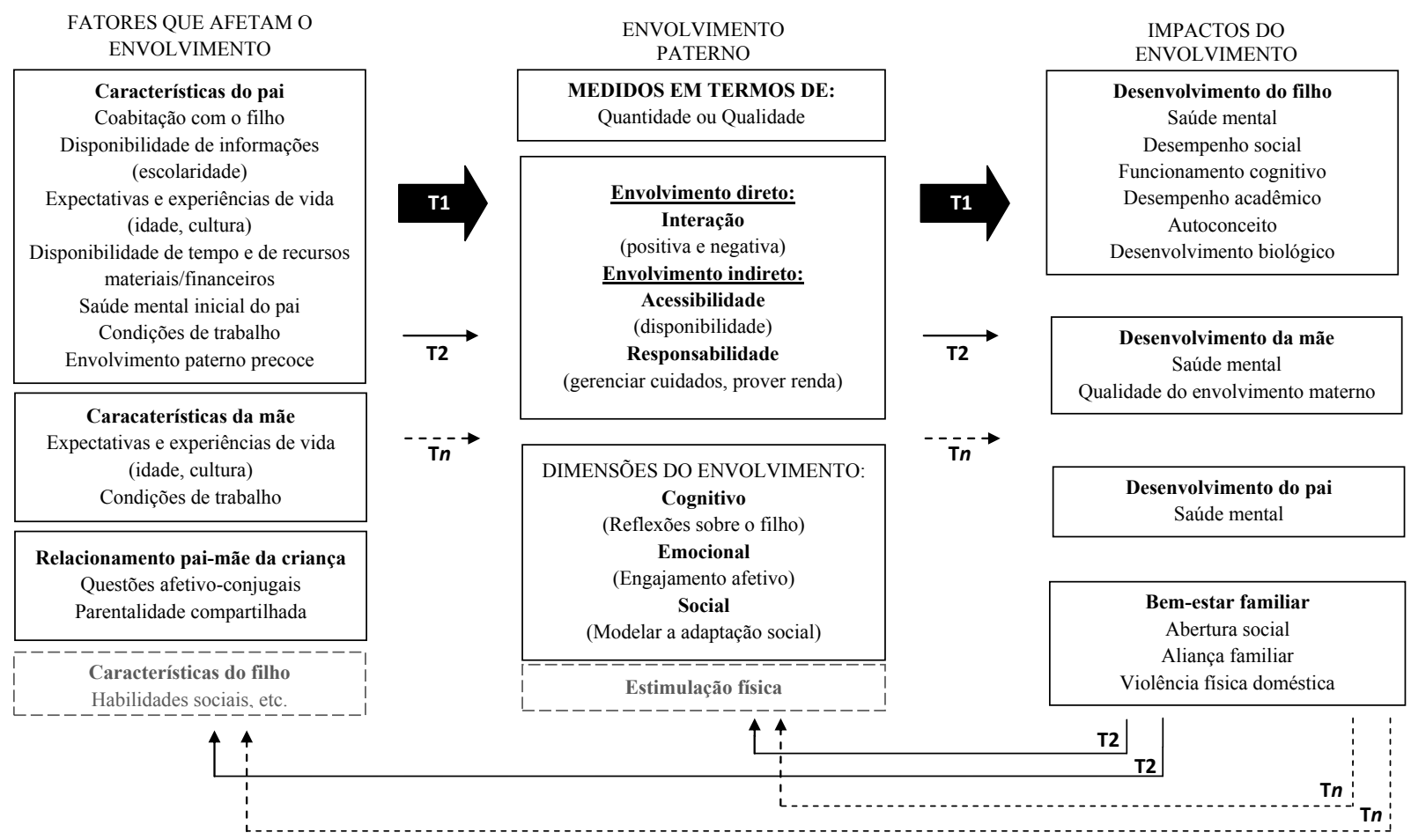

Figura 1. Modelo teórico de envolvimento paterno. Inicialmente (Tempo 1, T1), as condições dos pais afetam o envolvimento paterno, o que, por sua vez, resulta em determinados impactos. Em função dos impactos, ocorrem adaptações no T2, e assim sucessivamente (T1, T2 ...Tn).

autores (Cabrera, Shannon, \& Tamis-Lemonda, 2007; Volker, 2014). Sobre os impactos associados com a relação pai-filho, parece que o envolvimento paterno está associado com desfechos desenvolvimentais para os membros da família, com maior número de estudos investigando o desenvolvimento dos filhos, em consonância com outros autores que investigaram fatores que afetam o desenvolvimento infantil (Cabrera et al., 2007; Lamb, 2010; Volker, 2014).

Os resultados deste estudo e o modelo teórico proposto evidenciam, portanto, a relação entre o envolvimento paterno e outras vaiáveis, contribuindo para a identificação de correlatos importantes na elaboração de estudos futuros sobre o tema. À medida que profissionais são capazes de modificar condições, expectativas e habilidades parentais que afetam o envolvimento paterno, será possível testar a relação causal entre estes fatores e a qualidade do envolvimento do pai. Por sua vez, mais informações sobre os múltiplos impactos da relação pai-filho tornam mais visíveis os benefícios que podem ser alcançados por meio de programas de intervenção com pais. Além disso, a testagem empírica do modelo proposto, por meio de um procedimento pautado na abordagem dos Modelos de Equações Estruturais, poderá contribuir para a sua verificação e refinamento. O uso de um modelo teórico baseado em evidências é fundamental para a atuação de pesquisadores e profissionais da área da família que procuram aumentar a eficácia de intervenções, objetivando melhorar a qualidade de relações pai-filho.

\section{Referências}

*Alexander, A., O’Riordan, M. A., \& Furman, L. (2010). Do breastfeeding intentions of pregnant inner-city teens and adult women differ. Breastfeeding Medicine, 5(6), 289-296. doi:10.1089/bfm.2009.0083

Anfara, V. A., Brown, K. M., \& Mangione, T. L. (2002). Qualitative analysis on stage: Making the research process more public. Educational Researcher, 31, 28-38. doi:10.3102/0013189X031007028

*Bonner, M. J., Hardy, K. K., Willard, V. W., \& Hutchinson, K. C. (2007). Brief report: Psychosocial functioning of fathers as primary caregivers of pediatric oncology patients. 
Journal of Pediatric Psychology, 32(7), 851856. doi:10.1093/jpepsy/jsm011

*Brandão, S., \& Figueiredo, B. (2012). Fathers' emotional involvement with the neonate: Impact of the umbilical cord cutting experience. Journal of Advanced Nursing, 68(12), 2730-2739. doi:10.1111/j.1365-2648.2012.05978.x

*Broger, B., \& Zeni, M. B. (2011). Fathers' coping mechanisms related to parenting a chronically Ill child: Implications for advanced practice nurses. Journal of Pediatric Health Care, 25(2), 96104. doi:10.1016/j.pedhc.2009.09.004

*Bronte-Tinkew, J., Moore, K. A., Capps, R. C., \& Zaff, J. (2006). The influence of father involvement on youth risk behaviors among adolescents: A comparison of native-born and immigrant families. Social Science Research, 35, 181-209. doi:10.1016/j.ssresearch.2004.08.002

Byrne, B. M. (2010). Structural Equation Modeling with AMOS. Basic concepts, applications, and programmimng ( $2^{\text {nd }}$ ed.). New York: Routledge, Taylor \& Francis Group.

Cabrera, N. J., Shannon, J. D., \& Tamis-Lemonda, C. (2007). Father's influence on their children's cognitive and emotional development: From toddlers to pre-K. Applied Development Science, 11(4), 208-213. doi:10.1080/10888690701762100

*Cauce, A. M., Conger, R. D., Cruz, R. A., King, K. M., \& Widaman, K. F. (2011). Cultural influences on positive father involvement in two-parent Mexican-origin families. Journal of $\mathrm{Fa}$ mily Psychology, 25(5), 731-740. doi:10.1037/ a0025128

*Cerniglia, L., Cimino, S., \& Ballarotto, G. (2014). Mother-child and father-child interaction with their 24-month-old children during feeding, considering paternal involvement and the child's temperament in a community sample. Infant Mental Health Journal, 35(5), 473-481. doi:10.1002/imhj.21466

Cia, F. (2009). Um programa para aprimorar o envolvimento paterno: Impactos no desenvolvimento do filho (Tese de doutorado apresentado ao Programa de Pós-Graduação em Educação Especial, Universidade Federal de São Carlos, São Carlos, SP, Brasil).

*Cia, F., \& Barham, E. J. (2006). Influências das condições de trabalho do pai sobre o relacionamento pai-filho. Psico-USF, 11(2), 257-264. doi:10.1590/S1413-82712006000200014
*Cia, F., \& Barham, E. J. (2009). O envolvimento paterno e o desenvolvimento social de crianças iniciando as atividades escolares. Psicologia em Estudo, 14 (1), 67-74. doi:10.1590/S141373722009000100009

*Cia, F., Barham, E. J., \& Fontaine, A. M. G. V. (2010). Impactos de uma intervenção com pais: $\mathrm{O}$ desempenho acadêmico e comportamento das crianças na escola. Psicologia: Reflexão $e$ Crítica, 23(3), 533-543. doi:10.1590/S010279722010000300014

*Cia, F., Barham, E. J., \& Fontaine, A. M. G. V. (2012). Desempenho acadêmico e autoconceito de escolares: Contribuições do envolvimento paterno. Estudos de Psicologia (Campinas), 29(4),461470. doi:10.1590/S0103-166X2012000400001

*Dick, G. L. (2004). The Fatherhood Scale. Research on Social Work Practice, 14(2), 80-92. doi:10.1177/1049731503257863

*Ehrenberg, M. F., Gearing-Small, M., Hunter, M. A., \& Small, B. J. (2001). Childcare task division and shared parenting attitudes in dualearner families with young children. Family Relations, 50(2), 143-153. doi:10.1111/j.17413729.2001.00143.x

*Escribano, S., Aniorte, J., \& Orgilés, M. (2013). Factor structure and psychometric properties of the Spanish version of the Alabama Parenting Questionnaire (APQ) for children. Psicothema, 25 (3), 324-329.doi:10.7334/psicothema2012.315

*Fagan, J., \& Lee, Y. (2010). Perceptions and satisfaction with father involvement and adolescent mothers' postpartum depressive symptoms. Journal on Youth and Adolescence, 39, 11091121. doi:10.1007/s10964-009-9444-6

*Falceto, O. G., Fernandes, C. L., Baratojo, C., \& Giugliani, E. R. J. (2008). Factors associated with father involvement in infant care. Revista Saúde Pública, 42(6), 1-6. doi:10.1590/S003489102008000600009

*Falco, S. de, Esposito, G., Venuti, P., \& Bornstein, M. H. (2008). Fathers' play with their Down's syndrome children. Journal of Intellectual Disability Research, 52(6), 490-502. doi:10.1111/ j.1365-2788.2008.01052.x

*Finley, G. E., \& Schwartz, S. J. (2004). The Father Involvement and Nurturant Fathering Scales: Retrospective measures for adolescent and adult children. Educational and Psychological Measurement, 64 (1), 143-164. doi:10.1177/0013164403258453 
*Formoso, D., Gonzales, N. A., Barrera, M., Jr., \& Dumka, L. E. (2007). Interparental relations, maternal employment, and fathering in Mexican-American families. Journal of Marriage and the Family, 69, 26-39. doi:10.1111/j.17413737.2006.00341.x

*Frascarolo, F., Zaouche-Gaudron, C., \& Favez, N. (2005). Variations in fathers' discourse on fatherhood and in family alliances during infancy. European Journal of Psychology of Education, 20(2), 185-199. doi:10.1007/BF03173507

*Freeze, M. K., Burke, A., \& Vorster, A. C. (2014). The role of parental style in conduct disorders: A comparison between adolescent boys with and without conduct disorders. Journal of Child \& Adolescent Mental Health, 26(1), 63-73. doi:10. 2989/17280583.2013.865627

Gomes, L. B., Bossardi, C. N., Cruz, R. M., Crepaldi, M. A., \& Vieira, M. L. (2014). Propriedades psicométricas de instrumentos de avaliação do envolvimento paterno: revisão de literatura. Avaliação Psicológica, 13(1), 19-27. Recuperado em http://pepsic.bvsalud.org/pdf/avp/v13n1/ v13n1a04.pdf

*Gomez, R., \& Leal, I. (2007). Vinculação parental durante a gravidez: versão Portuguesa da Forma Materna e Paterna da Antenatal Emotional Attachment Scale. Psicologia, Saúde \& Doenças, 8 (2), 153-165. Retrieved from http:// www.scielo.mec.pt/pdf/psd/v8n2/v8n2a01.pdf

*Grzybowski, L. S., \& Wagner, A. (2010). O envolvimento parental após a separação/divórcio. Psicologia: Reflexão e Crítica, 23(2), 289-298. doi:10.1590/S0102-79722010000200011

*Habib, C., \& Lancaster, S. (2010). Changes in identity and paternal-foetal attachment across a first pregnancy. Journal of Reproductive and Infant Psychology, 28(2), 128-142. doi:10.1080/02646830903298723

*Halme, N., Tarkka, M. T., Paavilainen, E., Nummi, T., \& Åstedt-Kurki, P. (2010). The design and development of the father-child instrument (FCI) for assessing the characteristics of fathers availability and engagement with their preschool children. American Journal of Men's Health, 4(2), 145-156. doi:10.1177/1557988309331825

Kline, R. B. (2011). Principles and Practice of Structural Equation Modeling ( ${ }^{\text {rd }}$ ed.). New York: The Guilford Press.
Lamb, M. E. (1997). Father and child development: An introductory overview and guide. In M. E. Lamb (Ed.), The Role of the Father in Child Development (pp. 1-18). New York: John Wiley \& Sons.

Lamb, M. E. (2010). How do fathers influence children's development? Let me count the ways. In Lamb, M. E. (Ed.), The Role of the Father in Child Development ( $2^{\text {nd }}$ ed., pp. 1-26). Hoboken, NJ: John Wiley \& Sons.

*Ly, A. R., \& Goldberg, W. A. (2014). A new measure for fathers of children with developmental challenges. Journal of Intellectual Disability Research, 58(5), 471-484. doi:10.1111/jir.12044

Manfroi, E. C., Macarini, S. M., \& Vieira, M. L. (2011). Comportamento parental e o papel do pai no desenvolvimento infantil. Revista Brasileira Crescimento e Desenvolvimento Humano, 21(1): 59-69. Recuperado em http://pepsic. bvsalud.org/scielo.php?script=sci_arttext\&pid $=$ S0104-12822011000100007

Mehall, K. G., Spinrad, T. L., Eisenberg, N., \& Gaertner, B. M. (2009). Examining the relations of infant temperament and couples' marital satisfaction to mother and father involvement: A longitudinal study. Fathering, 7(1), 23-48. doi:10.3149/fth.0701.23

Murray, A. L., Rosengard, C., Weitzen, S., Raker, C. A., \& Phipps, M. G. (2012). Demographic and relationship predictors of paternity establishment for infants born to adolescent mothers. Pediatric and Adolescent Gynecology, 25, 322327. doi:10.1016/j.jpag.2012.05.015

Pasquali, L. (2010). Instrumentação psicológica: Fundamentos e práticas. Porto Alegre, RS: Artmed.

Piccinini, C. A., Silva, M. R., Gonçalves, T. R., Lopes, R. L., \& Tudge, J. (2004). O envolvimento paterno durante a gestação. Psicologia: Reflexão e Crítica, 17(3), pp.303-314. doi:10.1590/ S0102-79722004000300003

Pleck, J. H. (2007). Why could father involvement benefit children? Theoretical perspectives. Applied Development Science, 11(4), 196-202. doi: 10.1080/10888690701762068

Pleck, J.H.(2010). Paternal involvement: Revised conceptualization and theoretical linkages with child outcomes. In Lamb, M. E. (Ed.) The Role of the Father in Child Development (2a. ed.). Hoboken, New Jersey: John Wiley \& Sons, pp 28 a 58. 
*Ramchandani, P. G., Domoney, J., Sethna, V., Psychogiou, L., Vlachos, H., \& Murray, L. (2013). Do early father-infant interactions predict the onset of externalizing behaviors in young children? Findings from a longitudinal cohort study. Journal of Child Psychology and Psychiatry, 54(1), 56-64. doi:10.1111/j.14697610.2012.02583.x

*Salonna, F., Geckova, A. M., Zezula, I., Sleskova, M., Groothoff, J. W., Reijneveld, S. A., \& van Dijk, J. P. (2012). Does social support mediate or moderate socioeconomic differences in self-rated health among adolescents? International Journal of Public Health, 57, 609-617. doi:10.1007/s00038-011-0300-6

*Séjourné, N., Beaumé, M., Vaslot, V., \& Chabrol, H. (2012). Effect of paternity leave on maternal postpartum depression. Gynécologie Obstétrique \& Fertilité, 40, 360-364. doi:10.1016/j.gyobfe.2011.08.033

*Séjourné, N., Vaslot, V., Beaumé, M., Goutaudier, N., \& Chabrol, H. (2012). The impact of paternity leave and paternal involvement in childcare on maternal postpartum depression. Journal of Reproductive and Infant Psychology, 30(2), 135-144. doi:10.1080/02646838.2012.693155

*Shapiro, A. F., Krysik, J., \& Pennar, A. L. (2011). Who are the fathers in healthy families in Arizona? An examination of father data in at-risk families. American Journal of Orthopsychiatry, 81(3), 327-336. doi: 10.1111/j.19390025.2011.01101.x

*Silva, N. C. B., \& Aiello, A. R. L. (2009). Análise descritiva do pai da criança com deficiência mental. Estudos de Psicologia (Campinas), 26(4), 493 503. doi:10.1590/S0103-166X2009000400010

*Tessier, R., Charpak, N., Giron, M., Cristo, M., Calume, Z. F., \& Ruiz-Peláez, J. G. (2009). Kangoroo mother care, home environment and father involvement in the first year of life: A randomized controlled study. Acta Padiatrica, 98, 14441450. doi:10.1111/j.1651-2227.2009.01370.x
*Uji, M., Sakamoto, A., Adachi, K., \& Kitamura, T. (2014). The impact of authoritative, authoritarian, and permissive parenting styles on children's later mental health in Japan: Focusing on parent and child gender. Journal of Child and Family Studies, 23, 293-302. doi:10.1007/ s10826-013-9740-3

*Van Ee, E., Sleijpen, M., Kleber, R. J., \& Jongmans, M. J. (2013). Father-involvement in a refugee sample: Relations between posttraumatic stress and caregiving. Family Process, 52, 723-735. doi:10.1111/famp.12045

Volker, J. (2014). Paternal involvement: A review of the factors influencing father involvement and outcomes. TCNJ Journal of Student Scholarship, 16, 1-8. Retrieved from http://joss.pages. tcnj.edu/files/2014/04/2014-Volker.pdf

*Waller, M. R., \& Bitler, M. P. (2008). The link between couples' pregnancy intentions and behavior: Does it matter who is asked? Perspectives on Sexual and Reproductive Health, 40(4), 194-201. doi:10.1363/4019408

*Warpechowski, A., \& Mosmann, C. (2012). A experiência da paternidade frente à separação conjugal: sentimentos e percepções. Temas em Psicologia, 20(1), 247-260. Retrieved from http://pepsic.bvsalud.org/pdf/tp/v20n1/ v20n1a18.pdf

*Wysocki, T., \& Gavin, L. (2004). Psychometric properties of a new measure of fathers' involvement in the management of a pediatric chronic diseases. Journal of Pediatric Psychology, 29(3), 231-241. doi:10.1093/ jpepsy/jsh024 